\title{
Applications of Generalized Cascade Scattering Matrix on the Microwave Circuits and Antenna Arrays
}

\author{
Shun-Feng Cao, Yong-Chang Jiao, and Zheng Zhang \\ National Key Laboratory of Science and Technology on Antennas and Microwaves, Xidian University, Xian, Shaanxi 710071, China \\ Correspondence should be addressed to Shun-Feng Cao; shunfengcao@sina.cn
}

Received 9 December 2014; Revised 8 March 2015; Accepted 19 March 2015

Academic Editor: Angelo Liseno

Copyright ( 2015 Shun-Feng Cao et al. This is an open access article distributed under the Creative Commons Attribution License, which permits unrestricted use, distribution, and reproduction in any medium, provided the original work is properly cited.

\begin{abstract}
The ideal lossless symmetrical reciprocal network (ILSRN) is constructed and introduced to resolve the complex interconnections of two arbitrary microwave networks. By inserting the ILSRNs, the complex interconnections can be converted into the standard oneby-one case without changing the characteristics of the previous microwave networks. Based on the algorithm of the generalized cascade scattering matrix, a useful derivation on the excitation coefficients of antenna arrays is firstly proposed with consideration of the coupling effects. And then, the proposed techniques are applied on the microwave circuits and antenna arrays. Firstly, an improved magic-T is optimized, fabricated, and measured. Compared with the existing results, the prototype has a wider bandwidth, lower insertion loss, better return loss, isolation, and imbalances. Secondly, two typical linear waveguide slotted arrays are designed. Both the radiation patterns and scattering parameters at the input ports agree well with the desired goals. Finally, the feeding network of a two-element microstrip antenna array is optimized to decrease the mismatch at the input port, and a good impedance matching is successfully achieved.
\end{abstract}

\section{Introduction}

Microwave system consists of microwave elements and transmission lines, and the microwave network theory is one of the most important analytical approaches to design and synthesize the microwave equipment. A field analysis using Maxwell's equations is complete and rigorous. However, usually we are only interested in the terminal characteristics and the power flow through a device. And, sometimes, it is convenient to combine the elements together and find the responses without having to reanalyze the behavior of each element in combination with its neighbors. As a result, the complex and sensitive parts can be analyzed with a rigorous field analysis approach, while the characteristics of the entire network are obtained by microwave network theory.

Generally, a microwave network with an arbitrary number of ports can be characterized by the impedance, admittance, and scattering matrices. The transmission $(A B C D)$ matrix has been widely adopted in many applications, but it only works for two or more two-port networks. Recently, a generalized method for arbitrary networks was discussed by Hallbjörner [1]. It was based on the calculation of the sum of admittances by considering both the "internal" and "external" ports. At each intersection, the sum of admittances is obtained separately. And then, construct the scattering matrices of the elements and calculate the scattering matrix of the entire network according to the equations raised by the authors. In this paper, the principle is completely different, where the scattering matrices are cascaded oneby-one and based on different algorithms. The algorithm of generalized cascade scattering matrix has been presented in many researches and educational books [2-6]. However, it seems that the existing descriptions only present approaches to cope with the one-by-one interconnections. There is no distinct explanation on the complex interconnections. Besides, as far as we know, the algorithm is usually employed on microwave circuits, and it has never been applied on the excitation coefficients of antenna arrays.

In this paper, the algorithm of the generalized cascade scattering matrix is studied and expanded to deal with the 
complex interconnections and the excitation coefficients of antenna arrays. The ideal lossless symmetrical reciprocal network (ILSRN) is firstly presented to facilitate the generalized microwave cascade network. It can be treated as the "virtual joint" to combine the surrounding networks together. As a result, the generalized cascade scattering matrix can be expediently operated with an intuitive idea. The only thing one should to do is just to offer the interconnection relationships, and then the operations of the generalized cascade scattering matrices can be automatically performed on the computer. Moreover, a useful derivation on the excitation coefficients of antenna arrays is firstly proposed with consideration of the coupling effects. The antenna array as a whole is considered to be a network load of the feeding network. The excitation coefficients of each unit can be obtained through the interconnection of the feeding network and antenna array. To demonstrate the accuracy of the proposed techniques, applications both in the fields of microwave circuits and antenna arrays will be presented in the following.

The framework of this paper is as follows. In Section 2, the algorithm of the generalized cascade scattering matrix is derived, and the ILSRNs are illuminated in detail. Moreover, the derivation on the excitation coefficients of antenna arrays is presented. In Section 3, the proposed techniques are employed on several applications. Finally, we draw the conclusions in Section 4.

\section{Algorithms on the Generalized Cascade Scattering Matrix and Antenna Arrays}

Considering two arbitrary microwave networks I and II, the scattering matrices can be written as

$$
\begin{aligned}
& {\left[\begin{array}{l}
\mathbf{b}^{\mathrm{I}, \mathrm{R}} \\
\mathbf{b}^{\mathrm{I}, \mathrm{V}}
\end{array}\right]=\left[\begin{array}{ll}
\overline{\mathbf{S}}^{\mathrm{I}, \mathrm{R}} & \overline{\mathbf{S}}^{\mathrm{I}, \mathrm{V}} \\
\underline{\mathbf{s}}^{\mathrm{I}, \mathrm{R}} & \underline{\mathbf{s}}^{\mathrm{I}, \mathrm{V}}
\end{array}\right]\left[\begin{array}{l}
\mathbf{a}^{\mathrm{I}, \mathrm{R}} \\
\mathbf{a}^{\mathrm{I}, \mathrm{V}}
\end{array}\right]} \\
& {\left[\begin{array}{l}
\mathbf{b}^{\mathrm{II}, \mathrm{R}} \\
\mathbf{b}^{\mathrm{II}, \mathrm{V}}
\end{array}\right]=\left[\begin{array}{ll}
\overline{\mathbf{S}}^{\mathrm{II}, \mathrm{R}} & \overline{\mathbf{S}}^{\mathrm{II}, \mathrm{V}} \\
\underline{\mathbf{s}}^{\mathrm{II}, \mathrm{R}} & \underline{\mathbf{s}}^{\mathrm{II}, \mathrm{V}}
\end{array}\right]\left[\begin{array}{l}
\mathbf{a}^{\mathrm{II}, \mathrm{R}} \\
\mathbf{a}^{\mathrm{II}, \mathrm{V}}
\end{array}\right],}
\end{aligned}
$$

where the scattering matrices are separated into four cells related to the remaining $(\mathrm{R})$ and vanished $(\mathrm{V})$ ports, respectively. If the vanished ports of two different networks are interconnected one-by-one, they are satisfied by

$$
\begin{aligned}
\mathbf{b}^{\mathrm{I}, \mathrm{V}} & =\mathbf{a}^{\mathrm{II}, \mathrm{V}} \\
\mathbf{b}^{\mathrm{II}, \mathrm{V}} & =\mathbf{a}^{\mathrm{I}, \mathrm{V}} .
\end{aligned}
$$

Combining (1) and (2) yields the renewed scattering parameters at both the remaining and vanished ports

$$
\begin{aligned}
& {\left[\begin{array}{c}
\mathbf{b}^{\mathrm{I}, \mathrm{R}} \\
\mathbf{b}^{\mathrm{II}, \mathrm{R}}
\end{array}\right]=\left[\begin{array}{cc}
\overline{\mathbf{S}}^{\mathrm{I}, \mathrm{R}}+\overline{\mathbf{S}}^{\mathrm{I}, \mathrm{V}} \mathbf{K}_{2} \underline{\mathbf{S}}^{\mathrm{I}, \mathrm{R}} & \overline{\mathbf{S}}^{\mathrm{I}, \mathrm{V}} \mathbf{K}_{1} \underline{\mathbf{S}}^{\mathrm{I}, \mathrm{R}} \\
\overline{\mathbf{S}}^{\mathrm{II}, \mathrm{V}} \mathbf{K}_{4} \underline{\boldsymbol{S}}^{\mathrm{I}, \mathrm{R}} & \overline{\mathbf{S}}^{\mathrm{II}, \mathrm{R}}+\overline{\mathbf{S}}^{\mathrm{II}, \mathrm{V}} \mathbf{K}_{3} \underline{\mathbf{S}}^{\mathrm{II}, \mathrm{R}}
\end{array}\right]\left[\begin{array}{c}
\mathbf{a}^{\mathrm{I}, \mathrm{R}} \\
\mathbf{a}^{\mathrm{II}, \mathrm{R}}
\end{array}\right]} \\
& {\left[\begin{array}{c}
\mathbf{a}^{\mathrm{I}, \mathrm{V}} \\
\mathbf{a}^{\mathrm{II}, \mathrm{V}}
\end{array}\right]=\left[\begin{array}{ll}
\mathbf{K}_{2} \underline{\underline{\mathbf{S}}}^{\mathrm{I}, \mathrm{R}} & \mathbf{K}_{1} \underline{\boldsymbol{S}}^{\mathrm{II}, \mathrm{R}} \\
\mathbf{K}_{4} \underline{\boldsymbol{S}}^{\mathrm{I}, \mathrm{R}} & \mathbf{K}_{3} \underline{\mathbf{S}}^{\mathrm{II}, \mathrm{R}}
\end{array}\right]\left[\begin{array}{c}
\mathbf{a}^{\mathrm{I}, \mathrm{R}} \\
\mathbf{a}^{\mathrm{II}, \mathrm{R}}
\end{array}\right] .}
\end{aligned}
$$

Here, $\mathbf{K}_{1}, \mathbf{K}_{2}, \mathbf{K}_{3}$, and $\mathbf{K}_{4}$ are given by

$$
\mathbf{K}=\left[\begin{array}{cc}
\mathbf{E} & -\underline{\mathbf{S}}^{\mathrm{II}, \mathrm{V}} \\
-\underline{\mathbf{S}}^{\mathrm{I}, \mathrm{V}} & \mathbf{E}
\end{array}\right]^{-1}=\left[\begin{array}{ll}
\mathbf{K}_{1} & \mathbf{K}_{2} \\
\mathbf{K}_{3} & \mathbf{K}_{4}
\end{array}\right],
$$

where $\mathbf{E}$ is the unit matrix.

Note that the derivation is under a hypothesis that each pair of interconnection ports is interconnected oneby-one. Although similar conclusions have been drawn in many researches and educational books, none clearly indicate how to deal with the complex situations, for example, the interconnections with more than two ports and self-loops with the ports coming from the same network. Here, the ILSRNs are introduced to resolve the problem. Assume a three-port ILSRN has a generalized form of

$$
\mathbf{S}_{\text {ideal }}^{3}=\left[\begin{array}{lll}
S_{11} & S_{12} & S_{13} \\
S_{21} & S_{22} & S_{23} \\
S_{31} & S_{32} & S_{33}
\end{array}\right] .
$$

In terms of the reciprocity and symmetry, it generates

$$
\mathbf{S}_{\text {ideal }}^{3}=\left[\begin{array}{lll}
S_{11} & S_{12} & S_{12} \\
S_{12} & S_{11} & S_{12} \\
S_{12} & S_{12} & S_{11}
\end{array}\right] .
$$

Then, as to a lossless network, it is satisfied by

$$
\begin{aligned}
& S_{11}^{*} \cdot S_{11}+S_{12}^{*} \cdot S_{12}+S_{12}^{*} \cdot S_{12}=1, \\
& S_{11}^{*} \cdot S_{12}+S_{12}^{*} \cdot S_{11}+S_{12}^{*} \cdot S_{12}=0 .
\end{aligned}
$$

The above complex equation set contains four unknowns, and more conditions are needed to resolve it. At the threeport node, Kirchhoff's voltage law and current law can be applied to give

$$
\begin{aligned}
& V_{1}=V_{2} \\
& I_{1}+I_{2}+I_{3}=0,
\end{aligned}
$$

where $V_{i}$ and $I_{i}, i=1,2,3$, are the normalized voltage and current at the $i$ th port, respectively, and are defined by

$$
\begin{gathered}
V_{i}=a_{i}+b_{i} \\
I_{i}=a_{i}-b_{i} .
\end{gathered}
$$

Thus, this yields

$$
\begin{aligned}
& 1+S_{11}=S_{12} \\
& S_{11}+S_{12}+S_{12}=1 .
\end{aligned}
$$

From (8) and (11), the solutions can be found as

$$
\mathbf{S}_{\text {ideal }}^{3}=\frac{1}{3}\left[\begin{array}{ccc}
-1 & 2 & 2 \\
2 & -1 & 2 \\
2 & 2 & -1
\end{array}\right] .
$$




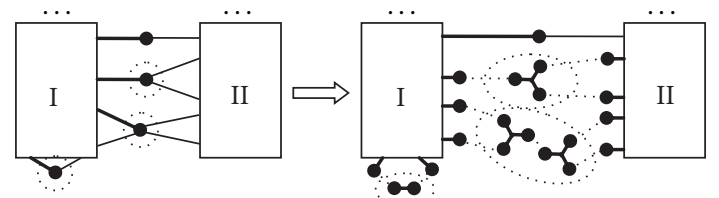

FIGURE 1: Decomposition of the complex interconnections.

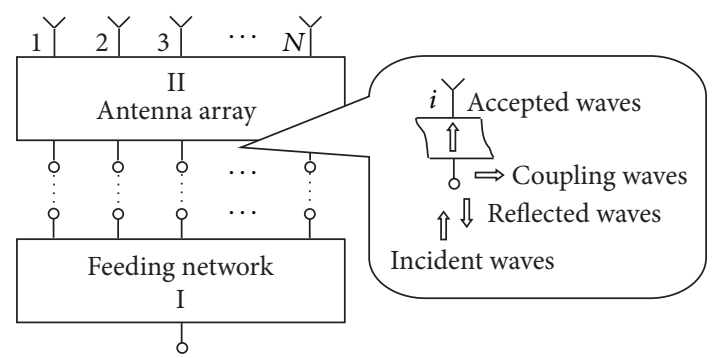

Figure 2: Interconnection between the feeding network and antenna array.

Similarly, the ILSRNs with an arbitrary number of ports can be constructed for particular problems. For example, the scattering matrices of two-port and four-port ILSRNs are given by

$$
S_{\text {ideal }}^{2}=\left|\begin{array}{ll}
0 & 1 \\
1 & 0
\end{array}\right|, \quad S_{\text {ideal }}^{4}=\frac{1}{2}\left|\begin{array}{cccc}
-1 & 1 & 1 & 1 \\
1 & -1 & 1 & 1 \\
1 & 1 & -1 & 1 \\
1 & 1 & 1 & -1
\end{array}\right| .
$$

To illuminate the decomposition of the complex interconnections, a sketch is shown in Figure 1. We find it contains three nonstandard interconnections (marked by circle lines). By inserting the two-port and three-port ILSRNs (marked by elliptical lines), extra virtual ports are introduced and the complex interconnections are converted into the standard one-by-one case as in the hypothesis without changing the network's characteristics. Also, we observe it is more advisable to replace the combination of two threeport ILSRNs by a single four-port ILSRN. Consequently, by inserting the ILSRNs, two arbitrary networks interconnected with arbitrary forms can be resolved with the standard algorithm as shown from (1) to (5). From (3), the scattering parameters at the remaining ports can be acquired. They contain the return loss, insertion loss, isolation, and phase distribution at each port, while the incident waves after the interconnection at the vanished ports can be obtained from (4). In this paper, just the incident waves will be utilized to calculate the excitation distribution of antenna elements when the antenna array is interconnected with a feeding network.

Antennas radiate electromagnetic waves towards the surrounding space with the coupling effects existing between the elements. As depicted in Figure 2, there are four kinds of energies at the $i$ th port, that is, incident waves, reflected waves, coupling waves, and accepted waves. According to the law of conservation of energy, the incident waves are split into the other three terms, where the reflected waves are returned into the feeding network, the coupling waves are transferred to the other elements, and the accepted waves are delivered to and consumed by the $i$ th element. They are satisfied by

$$
P_{\mathrm{inc}}^{i}=P_{\mathrm{ref}}^{i}+P_{\mathrm{cou}}^{i}+P_{\mathrm{acc}}^{i}
$$

where $P_{\mathrm{inc}}^{i}, P_{\mathrm{ref}}^{i}, P_{\mathrm{cou}}^{i}$, and $P_{\text {acc }}^{i}$ state the incident power, reflected power, coupling power, and accepted power at the $i$ th port, respectively. Thus, the accepted power can be obtained by

$$
\begin{aligned}
P_{\mathrm{acc}}^{i} & =P_{\mathrm{inc}}^{i}-P_{\mathrm{ref}}^{i}-P_{\mathrm{cou}}^{i} \\
& =P_{\mathrm{inc}}^{i}-P_{\mathrm{inc}}^{i} \cdot\left|S_{i i}\right|^{2}-P_{\mathrm{inc}}^{i} \cdot \sum_{j \neq i}\left|S_{j i}\right|^{2} \\
& =\frac{1}{2}\left|a_{i} \mathrm{II}, \mathrm{V}\right|^{2}\left(1-\sum_{j}\left|S_{j i}\right|^{2}\right) .
\end{aligned}
$$

In view of the phase factors of the incident waves $\overrightarrow{P_{\text {inc }}^{i}}=$ $\left.P_{\text {inc }}^{i} \angle a_{i}^{\mathrm{II}, \mathrm{V}}\right)$ and the input impedance of the $i$ th element $\left(\overrightarrow{P_{\mathrm{acc}}^{i}}=\right.$ $\left.(1 / 2)\left|I_{i}\right|^{2}\left|Z_{i}\right| \angle Z_{i}\right)$, the complex power delivered to the $i$ th element is

$$
\overrightarrow{P_{\mathrm{acc}}^{i}}=\frac{1}{2}\left|a_{i}^{\mathrm{II}, \mathrm{V}}\right|^{2}\left(1-\sum_{j}\left|S_{j i}\right|^{2}\right) \angle a_{i}^{\mathrm{II}, \mathrm{V}}=\frac{1}{2}\left|I_{i}\right|^{2}\left|Z_{i}\right| \angle Z_{i},
$$

where $\angle a_{i}^{\mathrm{II}, \mathrm{V}}$ and $\angle Z_{i}$ are the angles of the incident waves and the input impedance of the $i$ th element, respectively. In the array environment, suppose that the elements are similar and have the same radiation efficiencies and radiation impedances. Then, by considering the coupling effects, the complex amplitude excitation factor is given by

$$
\overrightarrow{I_{i}}=\left|a_{i}^{\mathrm{II}, \mathrm{V}}\right| \sqrt{\left(1-\sum_{j}\left|S_{j i}\right|^{2}\right)}\left(\angle a_{i}^{\mathrm{II}, \mathrm{V}}-\angle Z_{i}\right) .
$$

It should be noted that all the input ports have been already considered, and $a_{i}^{\mathrm{II}, \mathrm{V}}$ is obtained from (4) by considering the scattering matrices of both the feeding network and antenna array. Usually, all the elements are interconnected with the feeding network, and only one input port of the feeding network will be eventually remaining. In fact, the elements without excitations are terminated with open ends or matched loads, which can be treated as a part of the feeding network. In that case, it requires $\mathbf{a}^{\mathrm{I}, \mathrm{R}}=a_{\text {input }}$ and $\mathbf{a}^{\mathrm{II}, \mathrm{R}}=0$, and (4) will be reduced to

$$
\left[\begin{array}{c}
\mathbf{a}^{\mathrm{I}, \mathrm{V}} \\
\mathbf{a}^{\mathrm{II}, \mathrm{V}}
\end{array}\right]=\left[\begin{array}{c}
\mathbf{K}_{2} \underline{\mathbf{s}}^{\mathrm{I}, \mathrm{R}} \\
\mathbf{K}_{4} \underline{\mathbf{s}}^{\mathrm{I}, \mathrm{R}}
\end{array}\right] a_{\text {input }} .
$$


And then (17) can be expressed by

$$
\begin{aligned}
a_{i}^{\mathrm{II}, \mathrm{V}} & =\left(\sum_{k} \mathbf{K}_{4}^{i, k} \cdot \underline{\mathbf{S}}_{k, i}^{\mathrm{I}, \mathrm{R}}\right) \cdot a_{\text {input }} \\
I_{i} & =C\left|\sum_{k} \mathbf{K}_{4}^{i, k} \cdot \underline{\mathbf{S}}_{k, i}^{\mathrm{I}, \mathrm{R}}\right| \sqrt{1-\sum_{j}\left|S_{j i}\right|^{2}}\left(\angle a_{i}^{\mathrm{II}, \mathrm{V}}-\angle Z_{i}\right),
\end{aligned}
$$

where $C$ is a negligible constant.

A home-made program is developed in the environment of MATLAB to realize the interconnections of the generalized cascade scattering matrices. Firstly, the complex network is segmented into a set of ILSRNs and simple subnetworks, for example, short end, open end, matched load, transmission line, and transformer. The scattering matrices of these subnetworks can be easily acquired with the characteristic parameters. As to the complex and sensitive parts, they can be analyzed with commercial EM tools. Sometimes, these parts include various elements, and it is more advisable to measure them. And then, the simulated or measured matrices can be introduced into the network theory. As a result, by combining the network theory and a flexible method for the complex and sensitive parts, we can deal with most of microwave networks by the cascade scattering matrices. Secondly, all the ports are sequentially numbered, and then the interconnection relationships can be uniquely represented by a set of vectors. For instance, the vector of $\left[\begin{array}{llll}1 & 3 & 2 & 4\end{array}\right]$ may indicate the interconnections of 1-3 and 24 , where ports 1 and 2 come from a network while ports 3 and 4 come from another network. Thirdly, the scattering matrices of the ILSRNs and subnetworks together with the interconnection relationships are uploaded into the calculation procedure. Consequently, both the scattering parameters at the remaining ports and the excitation coefficients for antenna arrays can be obtained automatically by combining the subnetworks one-by-one.

The calculation procedure has its merits in two aspects: (1) the complicated operations are performed on the computer, which sometimes is impossible by manual work, especially for the networks with bigger sizes; (2) the calculation procedure is more time-saving compared with the conventional simulation methods, which can be embodied in the optimization algorithms to seek the optimum parameters.

\section{Applications}

3.1. An Improved Magic-T. Magic-T is a kind of microwave equipment with four ports and allows incident signals to be combined or subtracted with well-defined relative phases. Generally, the typical $3 \lambda / 2$ rat-race coupler has a narrowband frequency response. Using the microstrip-slotline transition, U-yen et al. presented a broadband magic-T by optimizing the physical parameters in the circuit simulation software $[7,8]$. We find the results are not good enough and there is still some room to improve the magic-T. Improvements have been done in two aspects: (1) the characteristic parameters, not the physical parameters, will be optimized

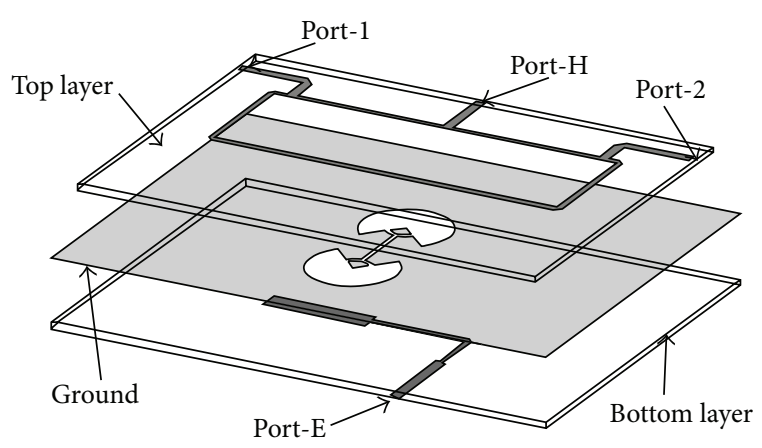

Figure 3: The explosive view of the magic-T.

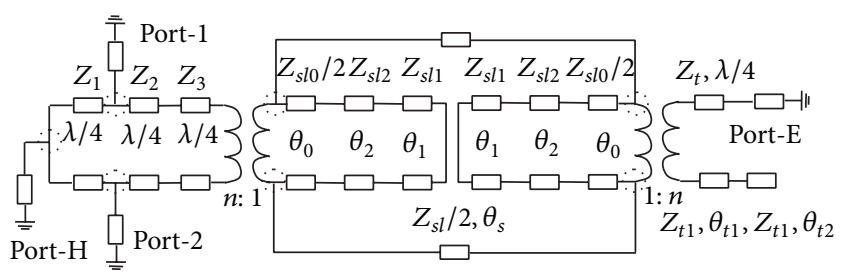

(a)

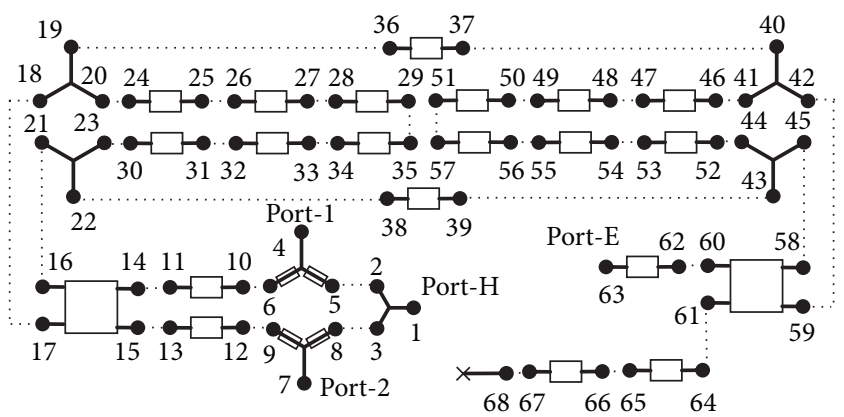

(b)

FIGURE 4: The circuit diagrams of the magic-T. (a) Equivalent circuit. (b) Decomposition circuit.

with the home-made hybrid genetic algorithm; (2) the $\mathrm{H}$ arm, port-1, and port- 2 are coplanerly placed on the top layer, while the E-arm is placed on the bottom layer to decrease the intensive coupling effects with other ports, as shown in Figure 3. The equivalent circuit and decomposition circuit of the improved magic-T are shown in Figure 4. It was found that there are seven nonstandard interconnections (marked by circle lines), which are replaced by the three-port ILSRNs (numbered by 1-2-3, 4-5-6, 7-8-9, 18-19-20, 21-22-23, 40-4142, and 43-44-45, resp.). Besides, there are two transformers (numbered by 14-15-16-17 and 58-59-60-61, resp.), an open end (numbered by 68), and a group of transmission lines.

The proposed techniques are adopted and the subnetworks are connected together. Numerical results indicate that the remaining ports will be the port-E (numbered by 63), port-H (numbered by 1 ), port-1 (numbered by 4 ), and port2 (numbered by 7 ). In addition, the time-cost is only 0.1 seconds, which makes it possible to have the calculation procedure embodied in the hybrid genetic algorithm to find 
optimum parameters satisfying the wideband demands [9]. The fitness function is defined by

Fitness

$$
=\frac{1}{\sum_{i}\left[\left|S_{\mathrm{EE}}\left(f_{i}\right)\right|^{2}+\left|S_{\mathrm{HH}}\left(f_{i}\right)\right|^{2}+\left|S_{11}\left(f_{i}\right)\right|^{2}+\left|S_{12}\left(f_{i}\right)\right|^{2}\right]},
$$

where $f_{i}$ is the observing frequencies ranging from $1 \mathrm{GHz}$ to $2 \mathrm{GHz}$ with steps of $0.05 \mathrm{GHz}$ and $S_{\mathrm{EE}}, S_{\mathrm{HH}}$, and $S_{11}$ represent the reflection coefficients at port-E, port- $\mathrm{H}$, and port-1, respectively, while $S_{12}$ is the isolation between port-1 and port-2. After 100 generations, the optimization procedure is terminated and the optimized parameters are shown as follows: $Z_{s l}=Z_{s l 0}=Z_{s l 2}=1.5, Z_{s l 1}=3.2, Z_{t}=1.2247, n_{1}=$ $n_{2}=1, Z_{1}=1.3333, Z_{2}=1.3968, Z_{3}=1.1905, \theta_{s}=9.111^{\circ}$, $\theta_{0}=9.397^{\circ}, \theta_{2}=6.5^{\circ}, \theta_{1}=30^{\circ}, Z_{t 1}=0.6, \theta_{t 1}=23.333^{\circ}$, $Z_{t 2}=0.5635$, and $\theta_{t 2}=31.587^{\circ}$. The optimized scattering parameters at the remaining ports are shown in Figure 5. It reveals that within a $40 \%$ bandwidth $(1.2 \mathrm{GHz}-1.8 \mathrm{GHz})$ the reflection coefficients at port- $\mathrm{H}$ are better than $-20 \mathrm{~dB}$, while there are a wider bandwidth and more excellent impedance matching at port-E and port-1. Ports 1-2 isolations are better than $25 \mathrm{~dB}$ from $1.23 \mathrm{GHz}$ to $1.74 \mathrm{GHz}$ and better than $20 \mathrm{~dB}$ from $1.15 \mathrm{GHz}$ to $1.81 \mathrm{GHz}$.

At $1.5 \mathrm{GHz}$, the optimized characteristic parameters are transformed into the physical dimensions, and the corresponding prototype is fabricated on the two-layer substrates as shown in Figure 6. The substrates are with the same relative dielectric constant of 6 and thickness of $1 \mathrm{~mm}$. The prototype is simulated with the software of Ansoft HFSS and measured using a network analyzer. The simulated and measured results are both shown in Figure 7. Compared with the results in Figure 5, we find the optimized, simulated, and measured results agree well with each other. The measured return losses are better than $20.8 \mathrm{~dB}(1.08 \mathrm{GHz}-1.82 \mathrm{GHz})$ at port1 and port-2, while the values arrived at $20.6 \mathrm{~dB}(1.18 \mathrm{GHz}-$ $1.86 \mathrm{GHz})$ and $20 \mathrm{~dB}(1.25 \mathrm{GHz}-1.82 \mathrm{GHz})$ at port-E and port- $\mathrm{H}$, respectively. It provides in-band insertion losses of $0.55 \mathrm{~dB}$ and $0.3 \mathrm{~dB}(1.2 \mathrm{GHz}-1.9 \mathrm{GHz})$ at port- $\mathrm{E}$ and port- $\mathrm{H}$, respectively. The difference is mainly due to the dissipated electromagnetic waves along the microstrip-slotline transition. Besides, the amplitude and phase imbalances are less than $0.25 \mathrm{~dB}$ and $1.5^{\circ}(1 \mathrm{GHz}-2 \mathrm{GHz})$ at port-E and port- $\mathrm{H}$, respectively. Ports $1-2$ and E-H isolations are better than $25 \mathrm{~dB}$ $(1.29 \mathrm{GHz}-1.85 \mathrm{GHz})$ and $34 \mathrm{~dB}(1 \mathrm{GHz}-2 \mathrm{GHz})$, respectively. Compared with the measured results in [8], our prototype has better frequency responses. In our work, within a similar relative bandwidth $(40 \%)$, the return losses are better than $10.6 \mathrm{~dB}, 10 \mathrm{~dB}, 10 \mathrm{~dB}$, and $11.8 \mathrm{~dB}$ at port-E, port- $\mathrm{H}$, port-1, and port-2, respectively, while ports 1-2 isolations are better than $10 \mathrm{~dB}$. In addition, the amplitude imbalance is one half of the result in [8], and the phase imbalance is slightly better in our work.

3.2. Waveguide Slotted Arrays. Waveguide slotted antennas are an important class of microwave antenna with numerous applications in radar and communication systems. Elliott

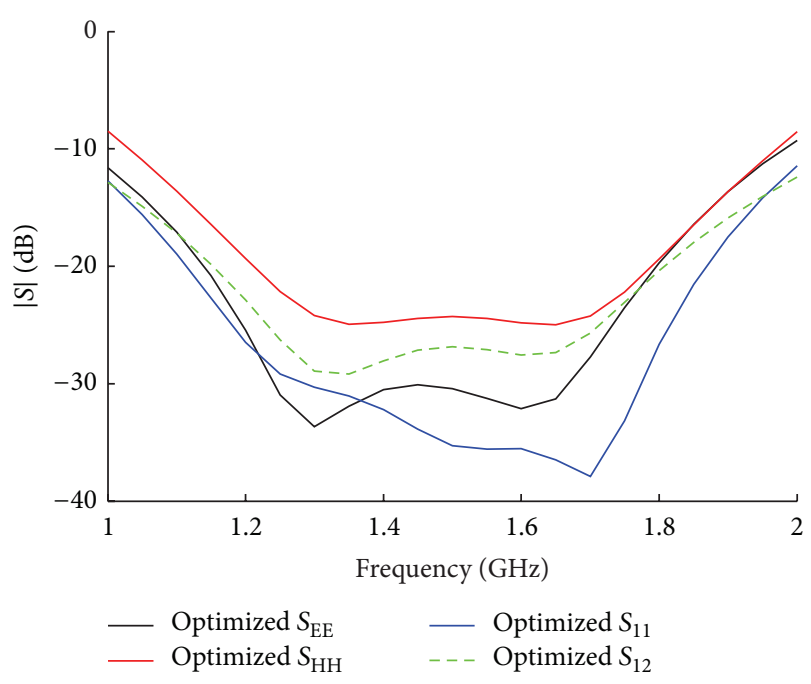

FIGURE 5: The optimized scattering parameters of the magic-T.

derived three famous formulas, which are still widely adopted nowadays [10-12]. Some computation methods were developed in [13-15]. The electromagnetic computation procedures operate well but usually are too sophisticated for most engineers. Morini et al. developed a strategy for the fullwave analysis of the waveguide slotted arrays, which is proved rigorously and able to deal with large arrays [16]. The core of the method is to segment the whole array into two regions: the "internal" and "external" one, where the internal one is connected together via generalized admittance matrices (GAMs), while the external one is analyzed by commercial or a home-made tool. The two regions are connected by "front plane," which is analyzed by the moment method in terms of modes of slots [16].

There are similarities and differences between our work and [16]. This work is based on the generalized cascade scattering matrix. The waveguide slotted array is naturally divided into two parts: the feeding network and slotted array. The main task is to find the scattering matrices of the two parts. The feeding network can be segmented into a set of ILSRNs and waveguide transmission lines. The ILSRNs are employed to connect the waveguide transmission lines together and offer extra ports for connecting the corresponding radiating slots. It is suitable for both the standard and nonstandard waveguides (e.g., ridge waveguides and waveguides filled with dielectric mediums). The scattering matrix of the entire waveguide feeding network can be obtained by connecting the decomposed subnetworks of ILSRNs and waveguide transmission lines. As to the slotted array, the impedance and admittance matrices are more convenient to get than the scattering matrices. The radiation characteristics of a longitudinal slot in the broad face are determined by the slot length and the offset from the center of the waveguide. In this paper, the self-admittance and resonant length of an isolated slot are extracted with the software of Ansoft HFSS. At a certain offset, by tuning the slot length to make the one-slot waveguide antenna resonant, the 


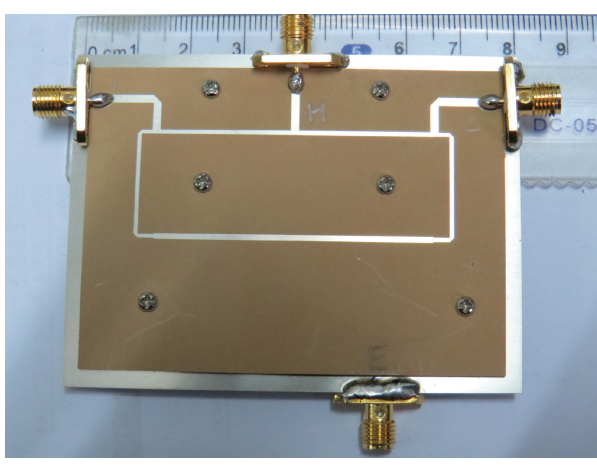

(a)

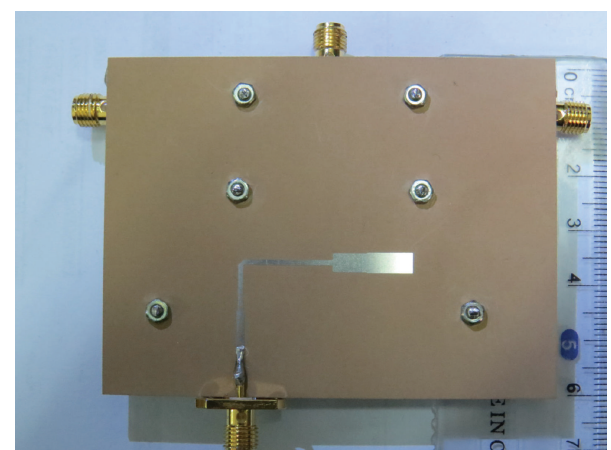

(b)

FIgURE 6: Photograph of the improved magic-T. (a) Top view. (b) Bottom view.

resonant self-admittance of the isolated slot can be acquired by

$$
Y_{\text {res }}=\frac{2}{S_{12}}-2 .
$$

It was found that the normalized admittances as a function of the slot length have similar variation trends for different offsets $[17,18]$. Thus the nonresonant self-admittances can be calculated via the interpolation method. As for the mutual impedances, the radiating slots are equivalent to the dipoles in terms of Babinet's principle [19]. As shown in Figure 8, according to the induced electromotive force method [20], the mutual impedances of two parallel side-by-side dipoles can be evaluated by

$$
\begin{aligned}
& Z_{12} \\
& =j 30\left\{\int_{h-l}^{h} \sin [k(l-h+z)]\right. \\
& \cdot\left[\frac{e^{-j k r_{1}}}{r_{1}}+\frac{e^{-j k r_{2}}}{r_{2}}-2 \cos (k l) \frac{e^{-j k r_{0}}}{r_{0}}\right] d z \\
& +\cdots \int_{h l}^{h+l} \sin [k(l+h-z)] \\
& \cdot\left[\frac{e^{-j k r_{1}}}{r_{1}}+\frac{e^{-j k r_{2}}}{r_{2}}\right. \\
& \left.\left.-2 \cos (k l) \frac{e^{-j k r_{0}}}{r_{0}}\right] d z\right\},
\end{aligned}
$$

where

$$
\begin{aligned}
& r_{0}=\sqrt{z^{2}+d^{2}} \\
& r_{1}=\sqrt{(z-l)^{2}+d^{2}} \\
& r_{2}=\sqrt{(z+l)^{2}+d^{2}} .
\end{aligned}
$$

In the following, two typical linear waveguide slotted arrays (resonant and traveling wave arrays) are presented to illuminate the principle of the proposed techniques. They are both operated at $10 \mathrm{GHz}$ with 12 slots and based on the standard rectangular waveguide of WR-90, which has an inside dimension of $22.86 \mathrm{~mm} \times 10.16 \mathrm{~mm}$ and a thickness of $1.27 \mathrm{~mm}$. The longitudinal round-head slots are located in the broad wall with a fixed width of $1.6 \mathrm{~mm}$. Once the offsets and lengths of the radiating slots are determined, the impedance matrix of the slotted array will be obtained in terms of the above-mentioned method. And then, the excitation distribution of the radiating slots can be acquired by connecting the scattering matrices of the feeding network and slotted array together. On the contrary, the offsets and lengths of the radiating slots can be synthesized with a desired excitation distribution, and they will be calculated according to the following steps.

Step 1. Initialize the offset and length of the last radiating slot.

Step 2. Find the offsets and lengths of the other radiating slots by minimizing the approximation errors of the calculated excitation distribution with respect to the desired one through optimization algorithms (e.g., genetic algorithm and hill-climbing algorithm).

Step 3. Check the calculated scattering parameters at the remaining ports. If the demands are not met, modify the offset and length of the last radiating slot and repeat Steps 2 and 3 .

Step 4. Stop.

3.2.1. Resonant Array. The schemes of the resonant array (spaced at $0.5 \lambda_{g}$ ) and its equivalent circuit are shown in Figure 9. The terminal short wall is replaced by a one-port network $(\mathbf{S}=-1)$. The aim of the array is to fulfill a $-30 \mathrm{~dB}$ peak side-lobe level (PSLL) with a $-30 \mathrm{~dB}$ Taylor illumination. According to the foregoing method, the slot parameters are obtained and listed in Table 1 . To verify the accuracy of the proposed algorithm, the corresponding prototype is simulated. The calculated and simulated radiation patterns in the yoz-plane are both shown in Figure 10. We observe that the results agree well with each other even at the high 


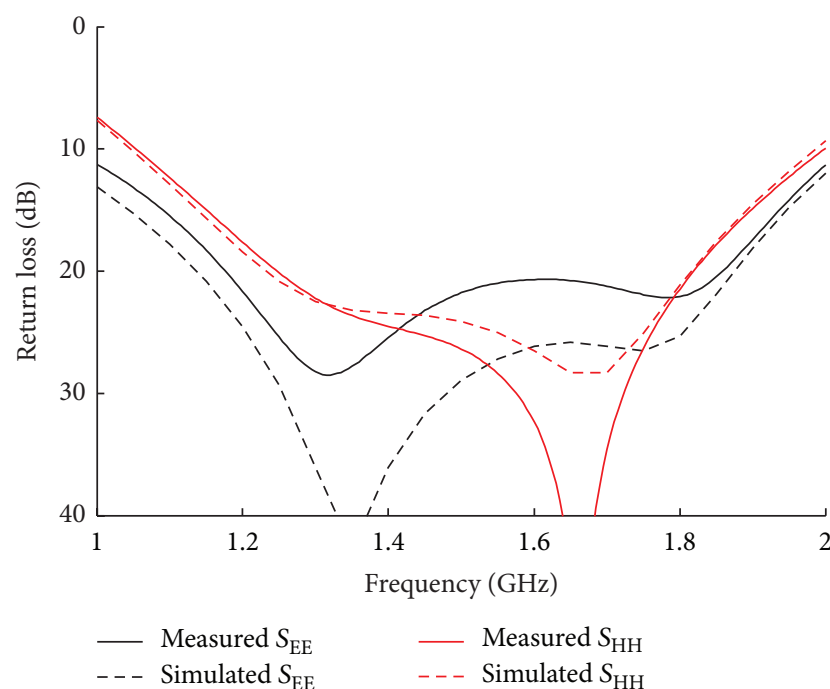

(a)

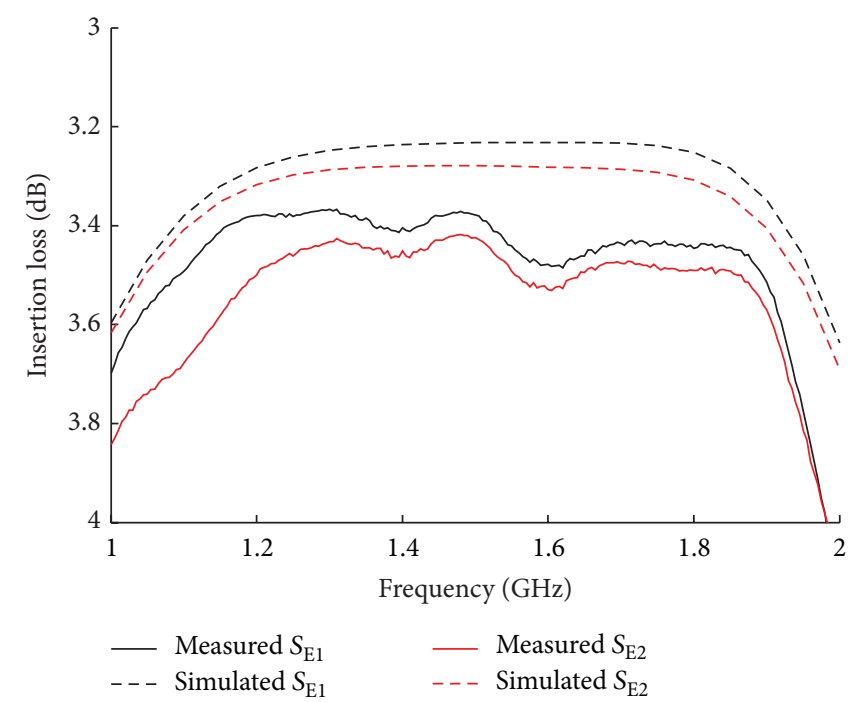

(c)

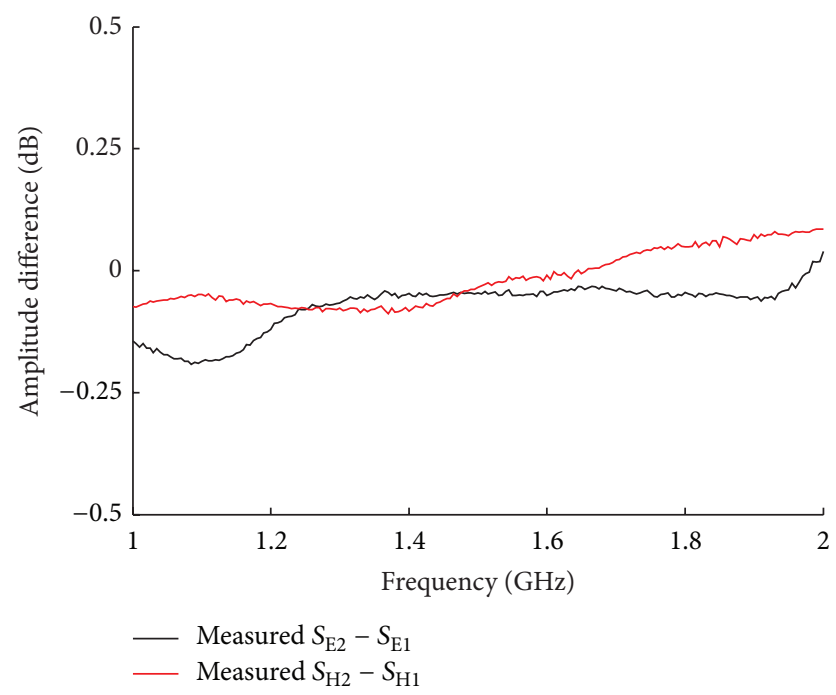

(e)

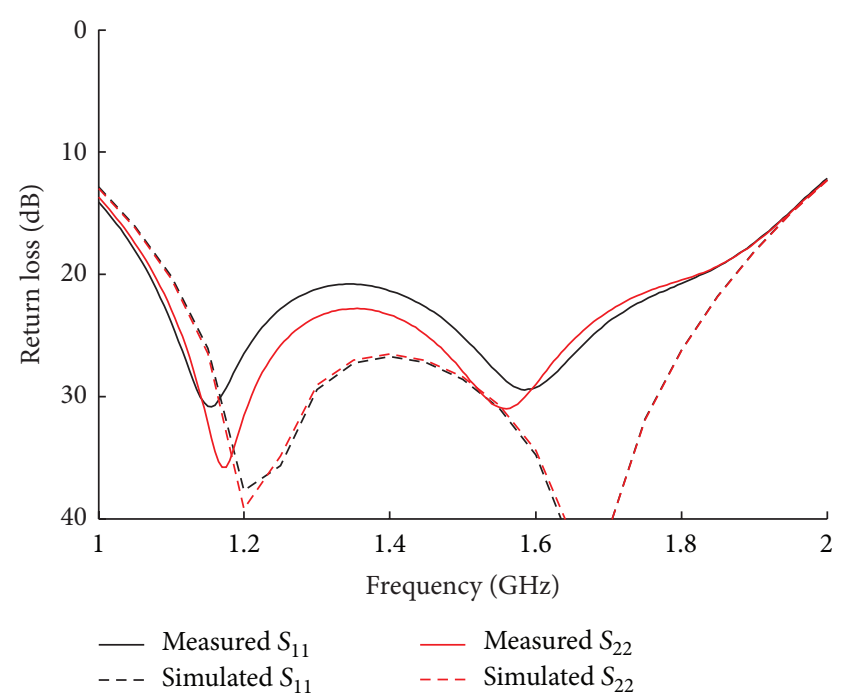

(b)

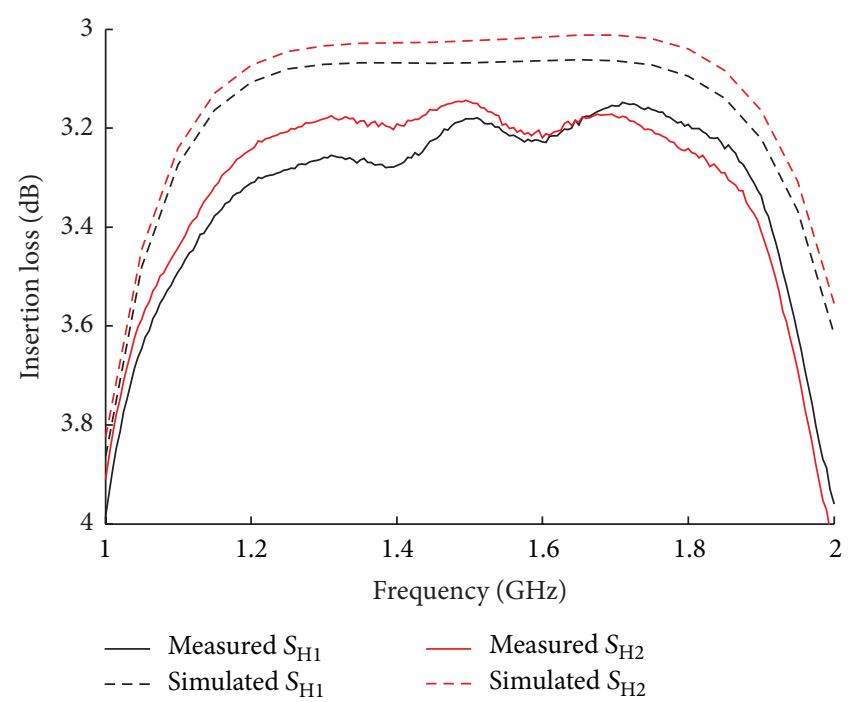

(d)

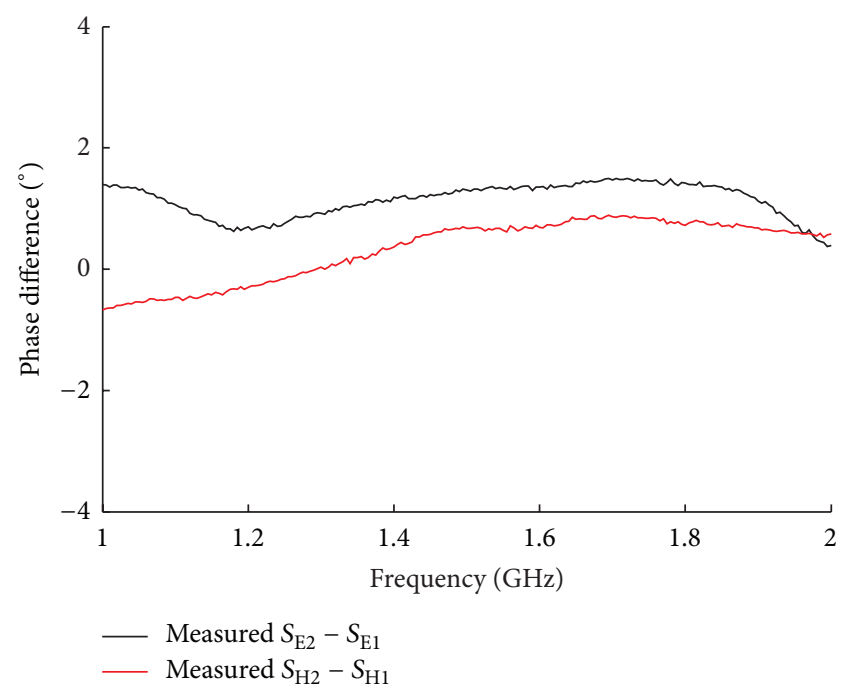

(f)

FIgURe 7: Continued. 


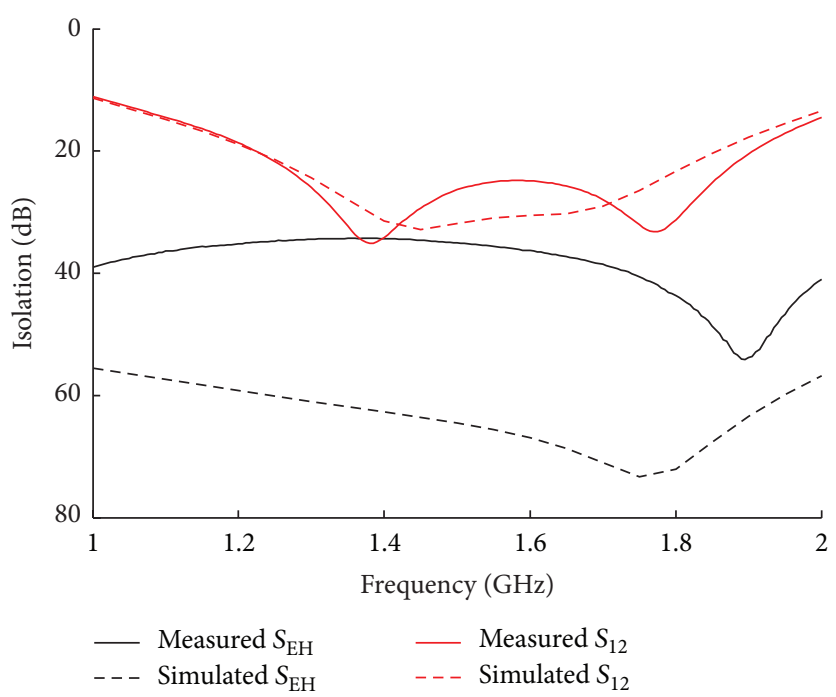

(g)

FIgURE 7: The results of the magic-T. (a) Return loss at port-E and port-H. (b) Return loss at port-1 and port-2. (c) Insertion loss at port-E-1 and port-E-2. (d) Insertion loss at port-H-1 and port-H-2. (e) Amplitude difference. (f) Phase difference. (g) Isolation at ports E-H and 1-2.

TABLE 1: Parameters of the resonant array.

\begin{tabular}{lcc}
\hline Element & Offset $(\mathrm{mm})$ & Length $(\mathrm{mm})$ \\
\hline 1 & 0.8701 & 14.4029 \\
2 & 1.2072 & 14.4239 \\
3 & 1.8556 & 14.4810 \\
4 & 2.5196 & 14.5580 \\
5 & 3.0715 & 14.6303 \\
6 & 3.3885 & 14.6727 \\
7 & 3.3837 & 14.6721 \\
8 & 3.0689 & 14.6285 \\
9 & 2.5131 & 14.5564 \\
10 & 1.8531 & 14.4807 \\
11 & 1.2068 & 14.4241 \\
12 & 0.8700 & 14.4029 \\
\hline
\end{tabular}

elevation angles. $\mathrm{A}-30 \mathrm{~dB}$ PSLL is achieved as expected. In addition, the calculated and simulated reflection coefficients at the input port are $-0.002-0.004 \mathrm{i}$ and $0.012-0.016 \mathrm{i}$ $(-33.9 \mathrm{~dB})$, respectively, which implies a good impedance matching at the input port.

The prototype is fabricated and connected with a coaxialto-waveguide transducer, as shown in Figure 11. The reflection coefficients and the radiation pattern in the yoz-plane at $10 \mathrm{GHz}$ are both measured, as seen in Figure 12. We observe that the measured results agreed well with the simulated or calculated ones. The measured reflection coefficients are better than $-15 \mathrm{~dB}$ from $9.82 \mathrm{GHz}$ to $10.21 \mathrm{GHz}$. At about $10 \mathrm{GHz}$, there is a small protuberance, which is mainly due to the coaxial-to-waveguide transducer. It has been proved that the reflection coefficients of the coaxial-to-waveguide transducer are better than $-20.7 \mathrm{~dB}$ from $8.2 \mathrm{GHz}$ to $12.4 \mathrm{GHz}$

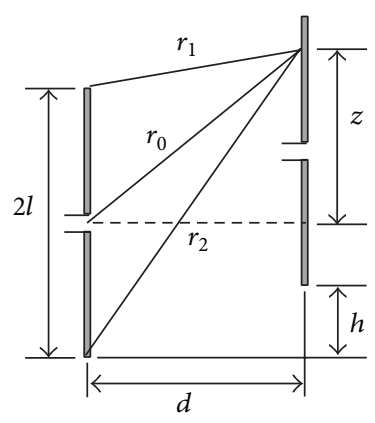

Figure 8: Two parallel dipoles.

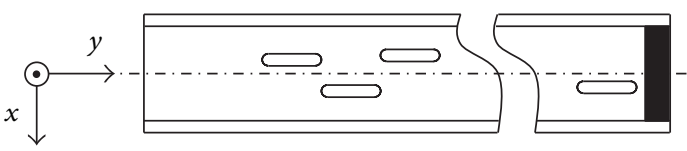

(a)

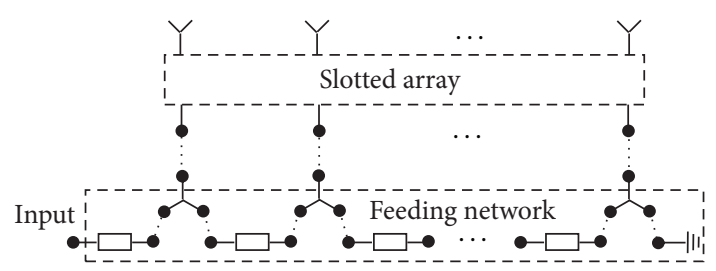

(b)

FIgure 9: The resonant array. (a) Configuration. (b) Equivalent circuit.

with the worst values around $10 \mathrm{GHz}$. In addition, we can find that the measured side-lobe levels are better than $-28.7 \mathrm{~dB}$, which is $1.3 \mathrm{~dB}$ higher than the desired value. 


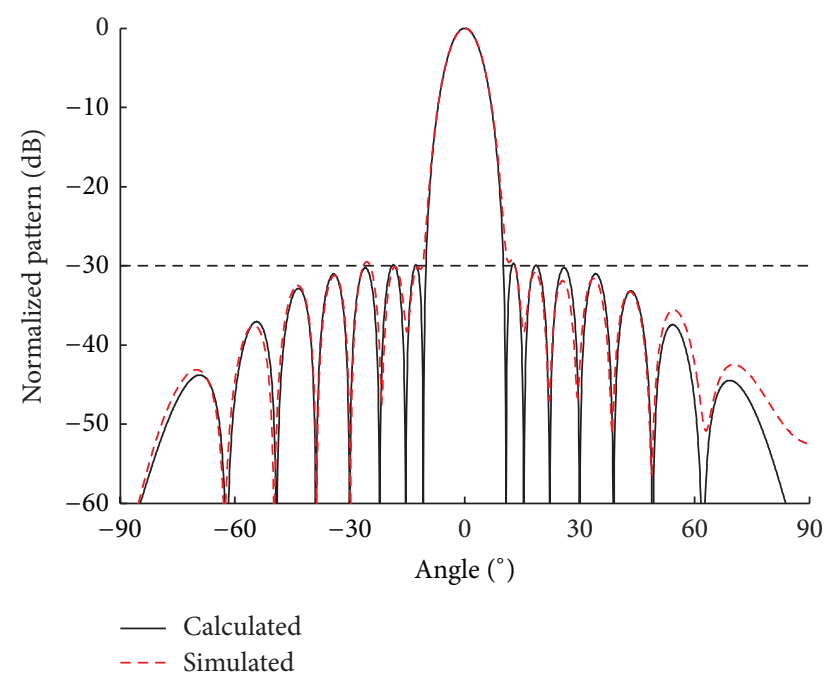

FIGURE 10: Radiation patterns of the resonant array.

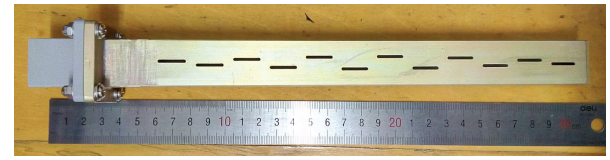

FIGURE 11: Photograph of the resonant waveguide slotted array.

3.2.2. Traveling Wave Array. The schemes of the traveling wave array (spaced at $0.6 \lambda_{q}$ ) and its equivalent circuit are shown in Figure 13. Unlike the resonant array, there is an extra port to monitor the absorbed power by the terminal matched load. The aim is to fulfill a $-25 \mathrm{~dB}$ PSLL $(-25 \mathrm{~dB}$ Taylor illumination) and lower absorbed power (higher efficiency). Eventually, the calculated parameters are listed in Table 2 while the corresponding calculated and simulated radiation patterns are shown in Figure 14. We observe that the results of the radiation patterns agree well with each other. It shows that the calculated reflection and transmission coefficients are $-0.006+0.008 \mathrm{i}$ and $-0.492+0.362 \mathrm{i}$, respectively, while the simulated ones are $-0.004+0.009 \mathrm{i}(-39.6 \mathrm{~dB})$ and $-0.462+$ $0.372 \mathrm{i}$ (an efficiency of $64.8 \%$ ), respectively. The additional demand of the efficiency is considered here and finally a good result is reached.

3.3. Microstrip Antenna Array. Without losing its generality, a two-element microstrip antenna array (spaced at $0.5 \lambda_{0}$ ) is shown in Figure 15. The substrate has a relative dielectric constant of 2.55 and thickness of $1 \mathrm{~mm}$. The antenna unit operates at $5.725 \mathrm{GHz}$ with the input impedance matched at $70 \Omega$ to avoid a large insertion length on the microstrip patch. The two-element array without the feeding network is simulated with the Ansoft HFSS and it shows that the scattering matrix (normalized at $70 \Omega$ ) at the two ports is

$$
S=\left[\begin{array}{cc}
-0.0364+0.1626 \mathrm{i} & -0.1664-0.1466 \mathrm{i} \\
-0.1664-0.1466 \mathrm{i} & -0.0364+0.1626 \mathrm{i}
\end{array}\right]
$$

TABLE 2: Parameters of the traveling wave array.

\begin{tabular}{lcc}
\hline Element & Offset $(\mathrm{mm})$ & Length $(\mathrm{mm})$ \\
\hline 1 & 0.9087 & 14.4148 \\
2 & 1.1343 & 14.4255 \\
3 & 1.5303 & 14.4579 \\
4 & 2.0086 & 14.5151 \\
5 & 2.4818 & 14.5847 \\
6 & 2.8906 & 14.6473 \\
7 & 3.1629 & 14.6845 \\
8 & 3.1962 & 14.6832 \\
9 & 2.9150 & 14.6402 \\
10 & 2.3943 & 14.5678 \\
11 & 1.8442 & 14.4934 \\
12 & 1.5000 & 14.4473 \\
\hline
\end{tabular}

In the conventional design, the feeding network, in fact a power divider, should be matched at $70 \Omega$ at the two antenna ports and matched at $50 \Omega$ at the input port of the feeding network. In that case, line 1 will be a one-fourth-wavelength transmission line with characteristic impedance of $59.2 \Omega$ to realize the impedance transformation from $50 \Omega$ to $70 \Omega$, while line 2 is utilized to connect the power divider and antenna array with characteristic impedance of $70 \Omega$. As a result, the reflection coefficient at the input port will arrive at $-12.1 \mathrm{~dB}$. The mismatch comes from two aspects, the coupling effects of the antenna units and the bad isolations of the feeding network.

Unlike the waveguide slotted arrays, the two-element array has a symmetrical configuration, and the excitation coefficients of antenna elements will be always equal. As a result, only the reflection coefficients are considered to decrease the mismatch at the input port. The equivalent circuit of the feeding network is constructed as shown in Figure 16. With the proposed techniques, the scattering matrices of the feeding network and the above simulated results of the two-element array are connected together, and the calculation process is embodied into a hill-climbing algorithm to optimize the characteristic impedances and electrical lengths of lines 1 and $2\left(Z_{1}, L_{1}, Z_{2}\right.$, and $\left.L_{2}\right)$ with $Z_{0}=50 \Omega$. Finally, the optimized parameters are shown as follows: $Z_{1}=62.82 \Omega, L_{1}=9.06 \mathrm{~mm}, W_{1}=1.934 \mathrm{~mm}$, $Z_{2}=46.96 \Omega, L_{2}=7 \mathrm{~mm}$, and $W_{2}=3.075 \mathrm{~mm}$. In terms of the optimized parameters, the feeding network is designed, and the entire prototype of the two-element array is simulated, fabricated, and measured. The photograph of the array and the results of the simulated and measured reflection coefficients are shown in Figures 17 and 18, respectively. The measured results show that the operation frequency has a slight excursion towards the lower frequencies. The main reason is that the relative dielectric constant of the substrate might be less accurate at such high frequencies. The reflection coefficient $(-24.8 \mathrm{~dB})$ has been improved greatly at the input port (normalized at $50 \Omega$ ) at $5.713 \mathrm{GHz}$. 


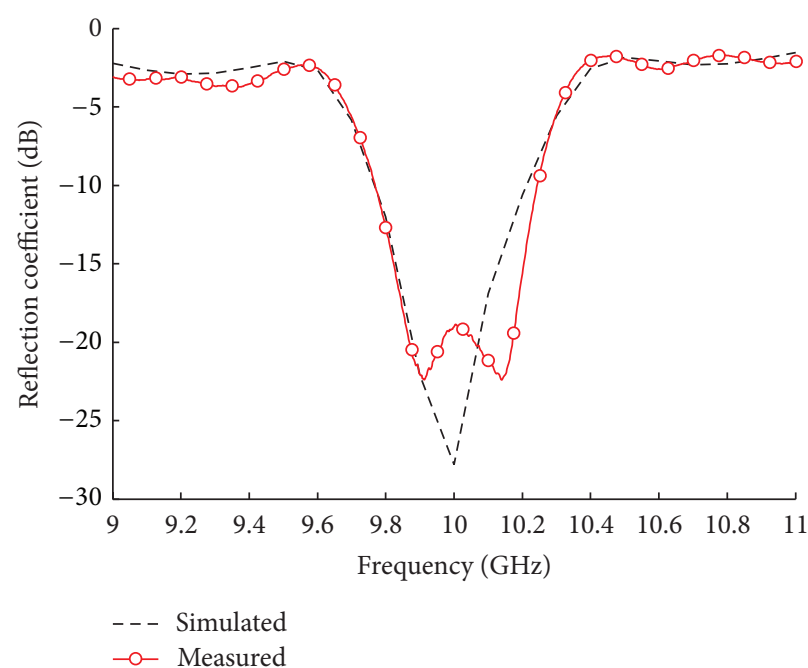

(a)

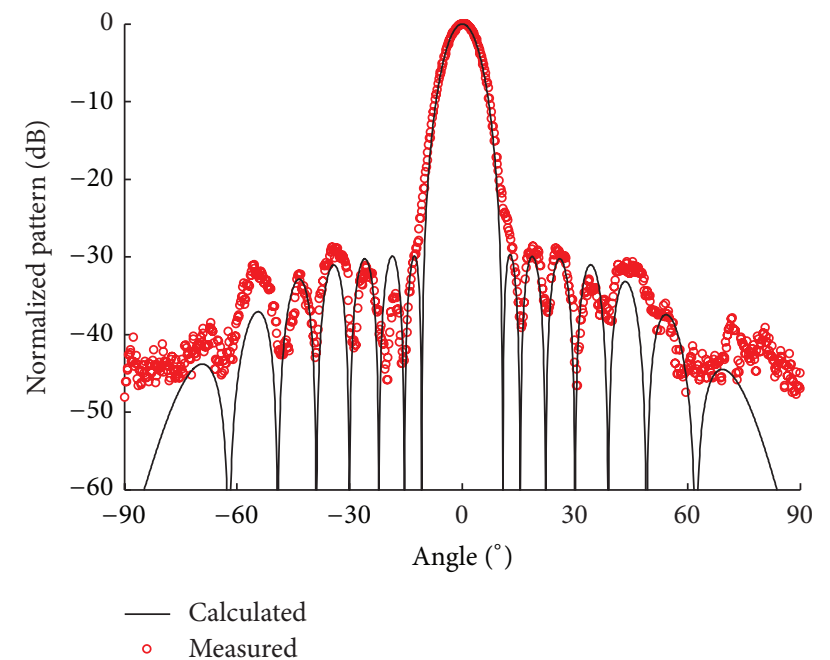

(b)

Figure 12: Measured results of the resonant waveguide slotted array. (a) Reflection coefficients. (b) Radiation pattern.

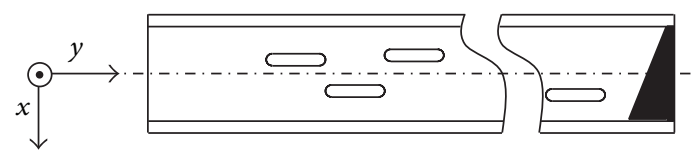

(a)

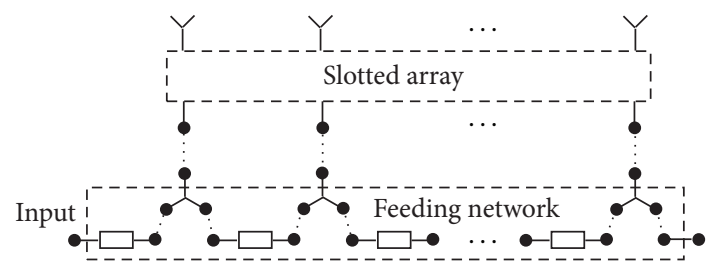

(b)

Figure 13: The traveling wave array. (a) Configuration. (b) Equivalent circuit.

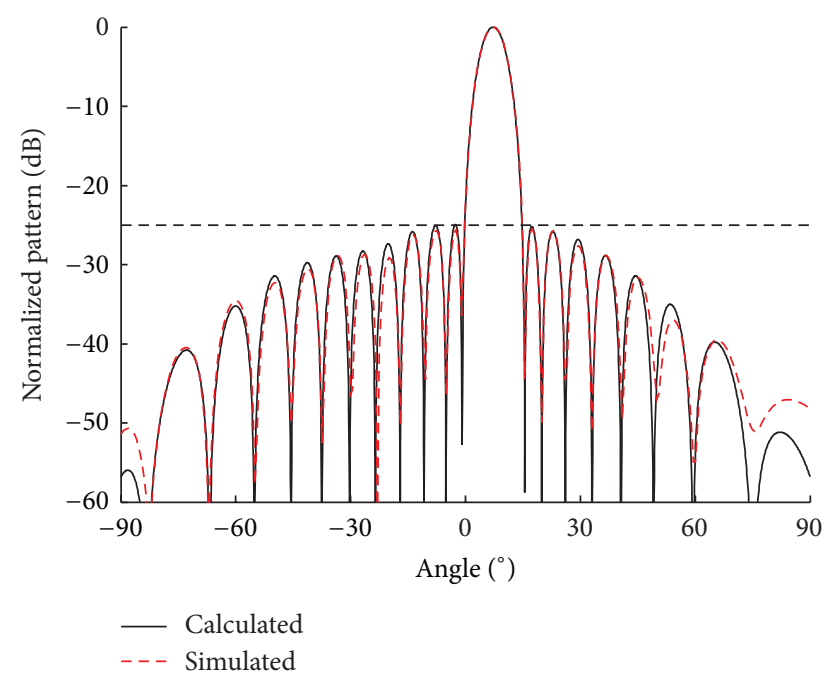

FIGURE 14: Radiation patterns of the traveling wave array.

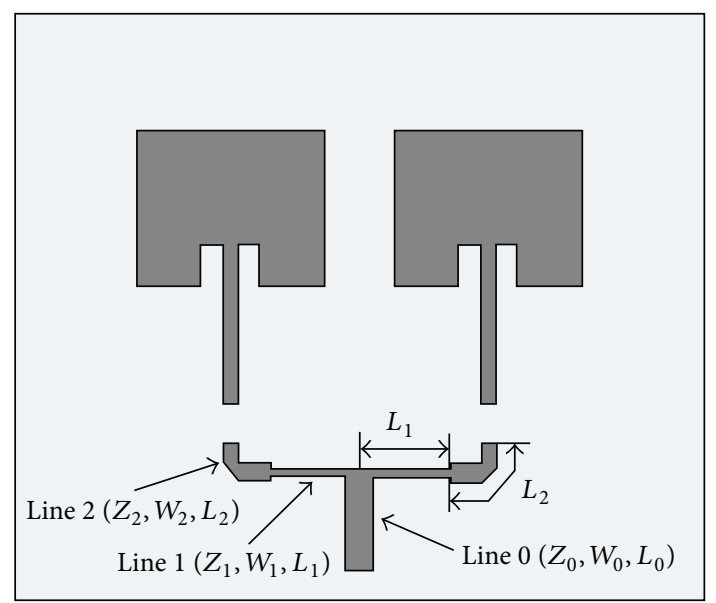

Figure 15: The configuration of the two-element array.

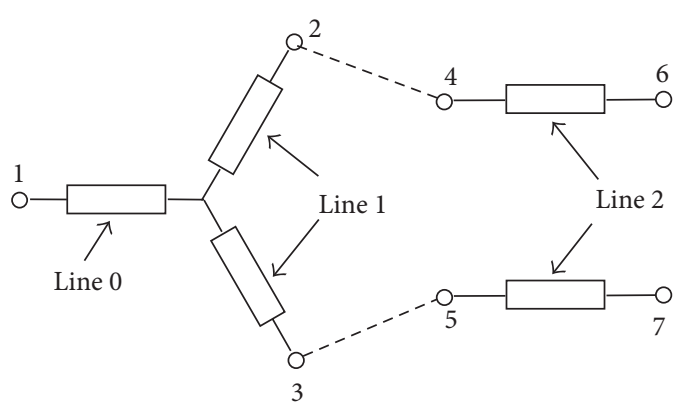

FIGURE 16: Equivalent circuit of the feeding network.

\section{Conclusion}

We present an approach to resolve the complex interconnections of two arbitrary microwave networks by introducing 


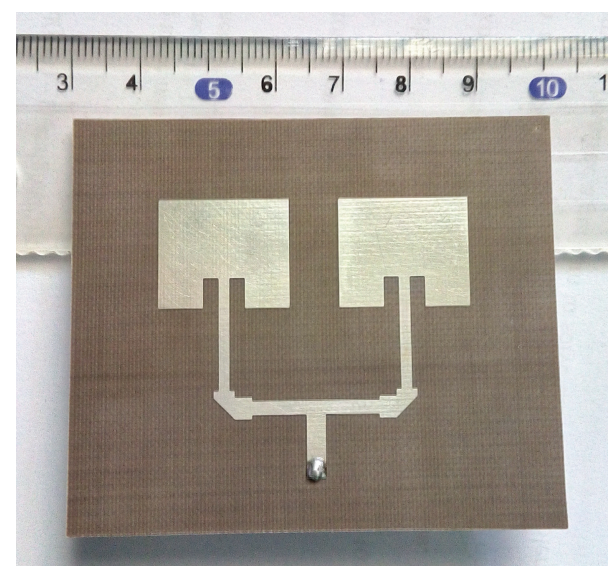

FIGURE 17: Photograph of the two-element array.

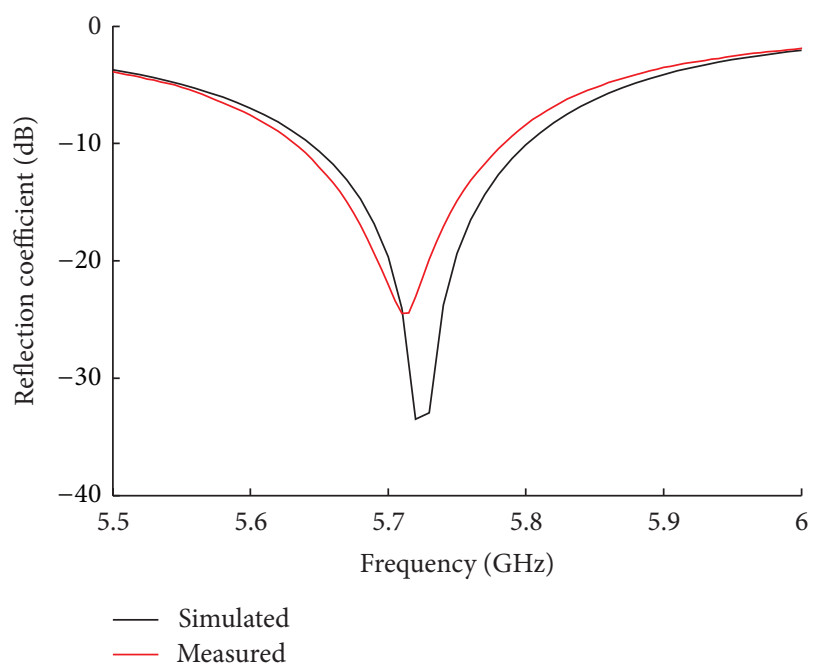

FIGURE 18: Reflection coefficients of the two-element array.

the ILSRNs. It greatly facilitates the operations of generalized microwave cascade network and is suitable for computeraided procedures. The algorithm of the generalized cascade scattering matrix is expanded to the field of antenna arrays. The excitation coefficients of antenna arrays are calculated according to the incident waves, which are obtained by connecting the scattering matrices of the feeding network and antenna arrays through the proposed techniques. Quantitative analysis of the excitation coefficients is available for antenna arrays with consideration of the coupling effects. As a result, the characteristics of the microwave circuits and antenna arrays can be accurately predicted, and advanced modifications can be adopted to rectify the undesired characteristics. The proposed techniques are verified by the applications on the microwave circuits and antenna arrays. And they have a promising prospect for many similar applications.

\section{Conflict of Interests}

The authors declare that there is no conflict of interests regarding the publication of this paper.

\section{References}

[1] P. Hallbjörner, "Method for calculating the scattering matrix of arbitrary microwave networks giving both internal and external scattering," Microwave and Optical Technology Letters, vol. 38, no. 2, pp. 99-102, 2003.

[2] D. M. Pozar, Microwave Engineering, John Wiley \& Sons, New York, NY, USA, 4th edition, 2011.

[3] J. A. Dobrowolski, Microwave Network Design Using the Scattering Matrix, Artech House, Boston, Mass, USA, 2010.

[4] G. Filipsson, "A new general computer algorithm for $S$-matrix calculation of interconnection multiports," in Proceedings of the 11th European Microwave Conference, pp. 700-704, Amsterdam, The Netherlands, September 1981.

[5] G. Figlia and F. Mercurio, "New design method for microwave cascade networks," in Proceedings of the Antennas and Propagation Society International Symposium-AP-S. Digest, pp. 946949, San Jose, Calif, USA, June 1989.

[6] B. A. Mishustin, "Synthesis of a reactive multiport network using a given scattering matrix," Izvestiya Vuz. Radiofizika, vol. 11, no. 12, pp. 1898-1906, 1968.

[7] K. U-yen, E. J. Wollack, S. Horst, T. Doiron, J. Papapol, and J. Laskar, "Slotline stepped circular rings for low-loss microstripto-slotline transitions," IEEE Microwave and Wireless Components Letters, vol. 17, no. 2, pp. 100-102, 2007.

[8] K. Uyen, E. J. Wollack, J. Papapolymerou et al., "Compact planar magic-T using microstrip-slotline transition," in Proceedings of the IEEE MTT-S International Microwave Symposium Digest, pp. 37-40, Honolulu, Hawaii, USA, 2007.

[9] J. H. Holland, Adaptation in Natural and Artificial Systems, MIT Press, Boston, Mass, USA, 1992.

[10] R. S. Elliott and L. A. Kurtz, "The design of small slot arrays," IEEE Transactions on Antennas and Propagation, vol. 26, no. 2, pp. 214-219, 1978.

[11] R. S. Elliott, "An improved design procedure for small arrays of shunt slots," IEEE Transactions on Antennas and Propagation, vol. 31, no. 1, pp. 48-53, 1983.

[12] R. S. Elliott and W. R. O'Loughlin, “The design of slot arrays including internal mutual coupling," IEEE Transactions on Antennas and Propagation, vol. 34, no. 9, pp. 1149-1154, 1986.

[13] G. A. Casula and G. Mazzarella, "Direct computation of the frequency response of planar waveguide slot arrays," IEEE Transactions on Antennas and Propagation, vol. 52, no. 7, pp. 1909-1912, 2004.

[14] K. Garb, R. Kastner, and R. Meyerova, "Analysis of planar arrays of shunt slots in ridged waveguides," Electronics Letters, vol. 30, no. 7, pp. 533-534, 1994.

[15] A. Enneking, R. Bayer, and F. Arndt, "Rigorous analysis of large finite waveguide-fed slot arrays including the mutual internal and external higher-order mode coupling," in Proceedings of the IEEE Antennas and Propagation Society International Symposium (AP-S '00), pp. 74-77, Salt Lake City, Utah, USA, July 2000.

[16] A. Morini, T. Rozzi, and G. Venanzoni, "On the analysis of slotted waveguide arrays," IEEE Transactions on Antennas and Propagation, vol. 54, no. 7, pp. 2016-2021, 2006. 
[17] L. G. Josefsson, "Analysis of longitudinal slots in rectangular waveguides," IEEE Transactions on Antennas and Propagation, vol. 35, no. 12, pp. 1351-1357, 1987.

[18] H. Matzner and N. Amir, "Efficient moment method solution for a longitudinal slot in a rectangular waveguide," IEE Proceedings-Microwaves, Antennas and Propagation, vol. 153, no. 4, pp. 335-340, 2006.

[19] S. Silver, Microwave Antenna Theory and Design, McGraw-Hill, New York, NY, USA, 1949.

[20] J. D. Kraus and R. J. Marhefka, Antennas: For All Applications, McGraw-Hill, New York, NY, USA, 3rd edition, 1997. 

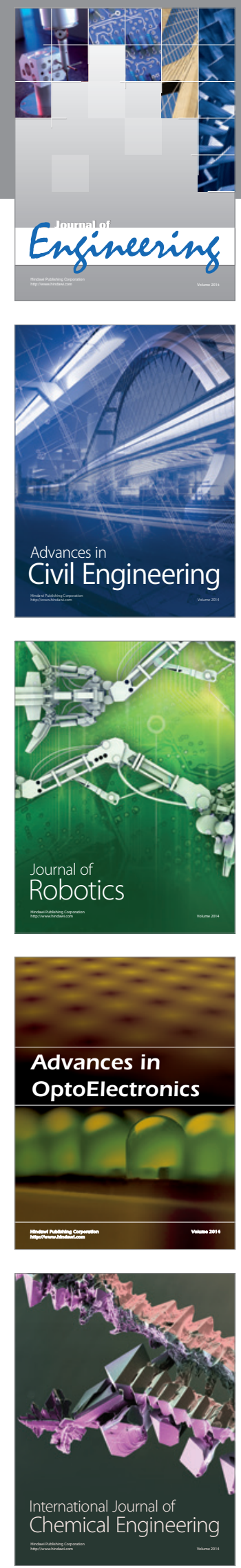

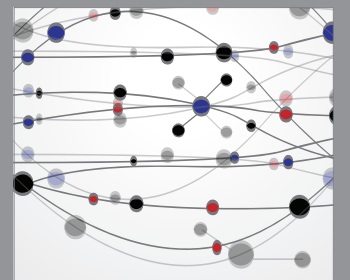

The Scientific World Journal
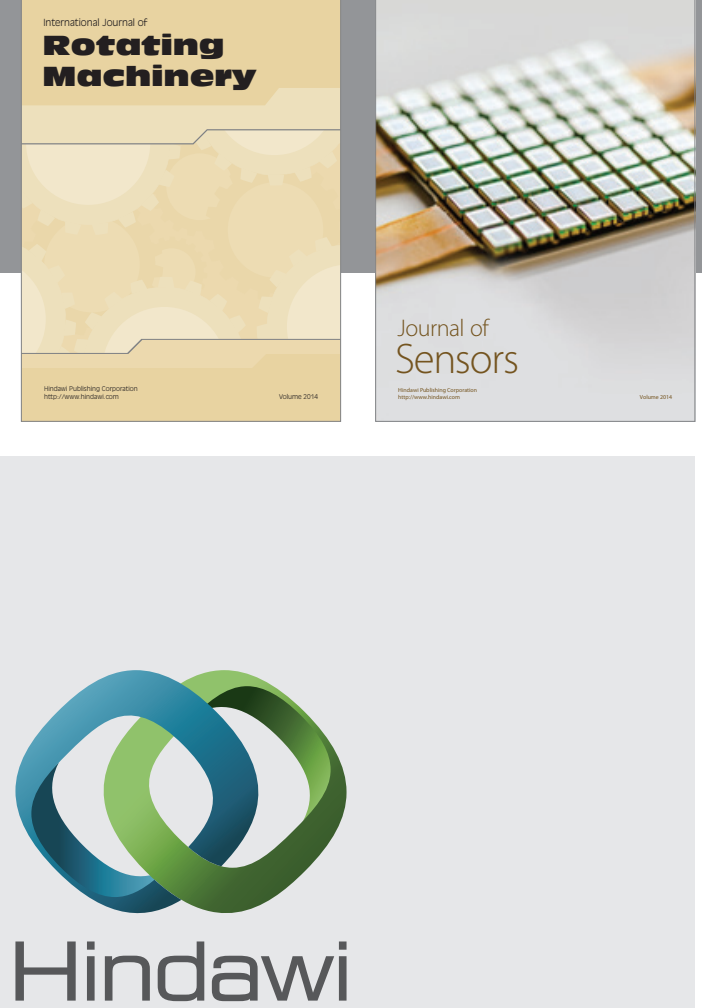

Submit your manuscripts at http://www.hindawi.com
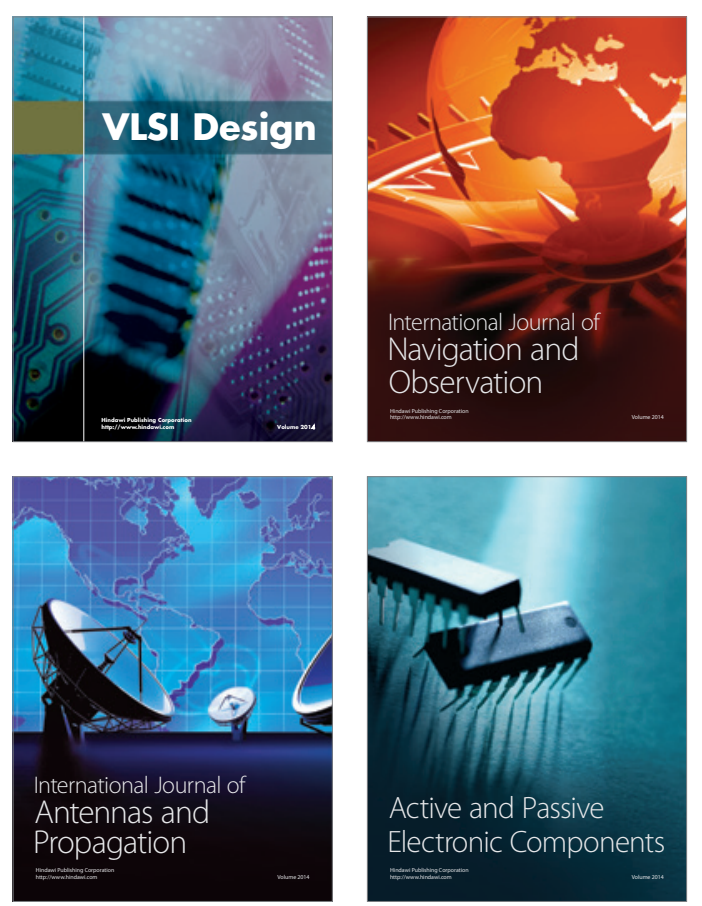
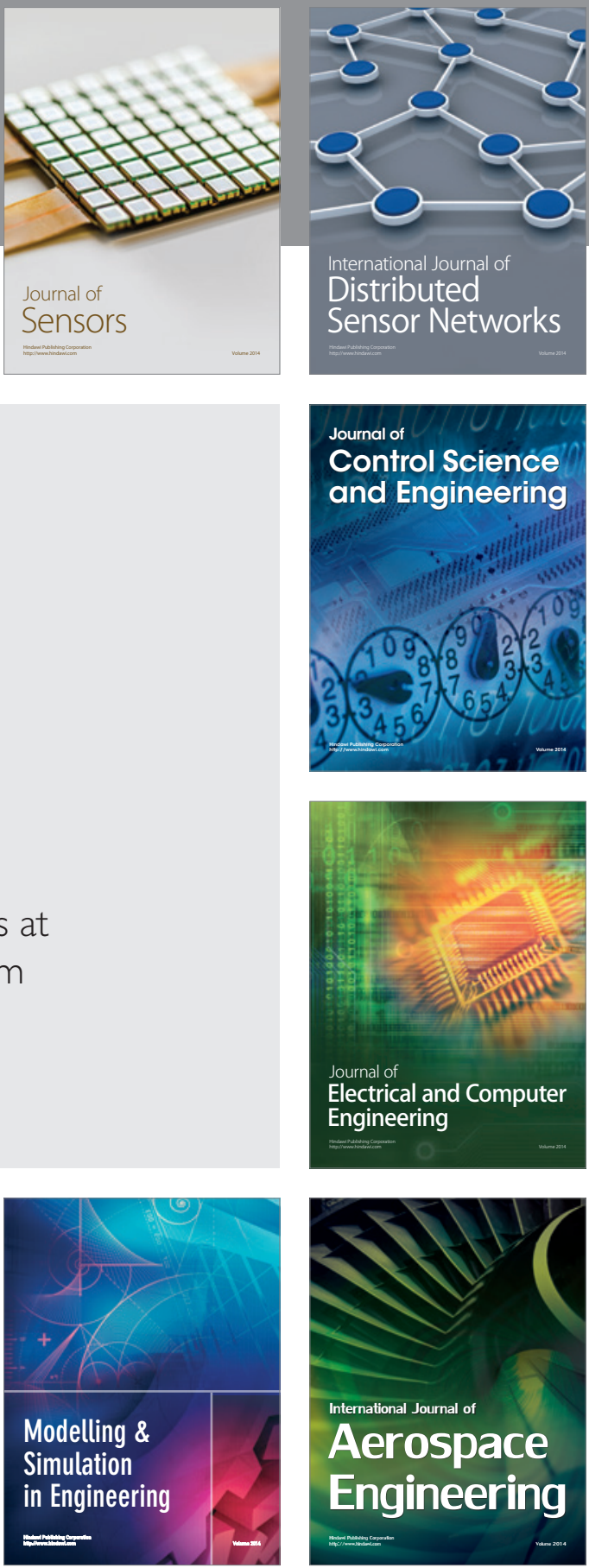

Journal of

Control Science

and Engineering
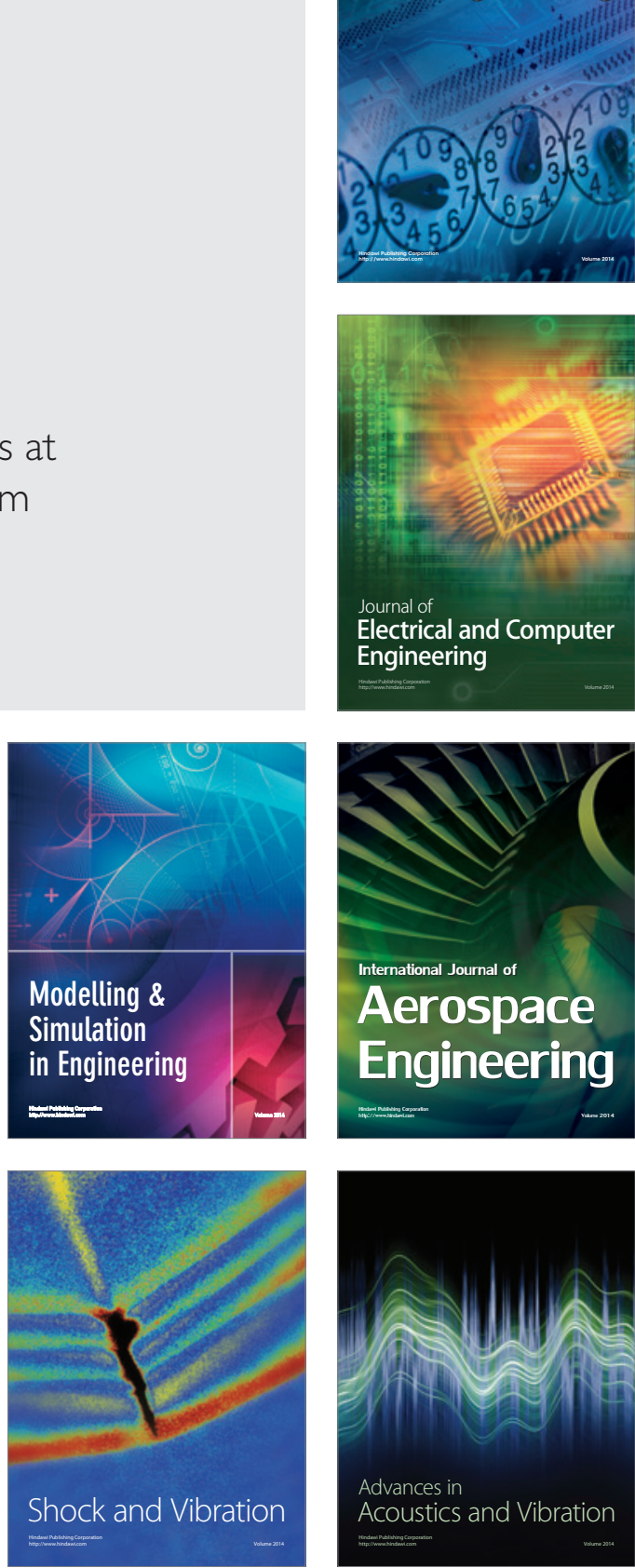